\title{
Top quark production in ATLAS
}

\author{
C. Bertella*t \\ CPPM, Centre de Physique des Particules de Marseille \\ E-mail: bertelladcern.ch
}

Measurements of the top quark production cross sections in proton-proton collisions with the ATLAS detector at the Large Hadron Collider are presented. The measurement requires no, one or two electrons or muons in the final state (single lepton, dilepton, hadronic channel). The main focus are measurements of differential spectra of ttbar final states, in particular, measurements that are able to constrain the modelling of additional parton radiation. We also discuss the production of top quark pairs in association with heavy quarks (beauty and charm). Measurements of single top-quark production in the $\mathrm{t}$ - and $\mathrm{Wt}$-channels are presented and determination of the CKM matrix element $\left|V_{t b}\right|$ is discussed.

XXI International Workshop on Deep-Inelastic Scattering and Related Subject -DIS2013, 22-26 April 2013

Marseilles, France

\footnotetext{
* Speaker.

† On behalf of ATLAS Collaboration.
} 


\section{Introduction}

The top quark is the heaviest fundamental particle, discovered in 1995 by the CDF [1] and D0 [2] Collaborations. Precision measurements of the top quark production cross section and properties give insight on perturbative QCD and on processes that eventually represent important sources of background in several new physics scenarios. Furthermore, thanks to its Yukawa coupling, the top quark might shed light on the electroweak symmetry breaking mechanism. At the LHC the top quark is produced via strong interaction leading to top-anti top final states or via weak interaction leading to single top in the final state. Two mechanisms contribute to the top pair production: gluon fusion and quark-anti quark annihilation. On the other hand, single top production occurs via the exchange of a virtual $W$ boson (either in the $t$ - and $s$-channels) or in association with an on-shell $W$ boson.

\section{Top pair production}

The most recent theoretical prediction on the total cross-section for the production of top quark pairs relies on next-to-next-to-leading (NNLO) and next-to-next-to-leading-logarithmic (NNLL) order calculations and reports a value of $172.0_{-5.8-4.8}^{+4.4+4.7} \mathrm{pb}\left(245.8_{-8.4-6.4}^{+6.2+6.2} \mathrm{pb}\right)$ for a centre-of-mass energy of $\sqrt{s}=7 \mathrm{TeV}(8 \mathrm{TeV})[3]$. Measurements of $\sigma_{t \bar{t}}$ have been performed in all decay modes, which are classified according to the decay of the $W$-bosons.

In this paper single, dilepton (where lepton is either an electron or a muon) and all-hadronic final states have been considered. The $t \bar{t}$ cross section result presented by ATLAS [4] combines those final states and finds a value of $\sigma_{t \bar{t}}=177 \pm 3$ (stat. $)_{-7}^{+8}$ (syst.) \pm 7 (lumi.) pb at $\sqrt{s}=7 \mathrm{TeV}$, which is in good agreement with the SM expectation.

\subsection{Semileptonic channel}

The most precise measurement of the $t \bar{t}$ cross section is provided by the single lepton channel analysis. The channel have been investigated at $\sqrt{s}=7 \mathrm{TeV}$ and $8 \mathrm{TeV}$; here just the most recent results performed at $\sqrt{s}=8 \mathrm{TeV}$ has been considered. The main background processes are $W+\mathrm{jets}$ and QCD multi-jet events, where one jet is mis-identified as a lepton. A likelihood discriminant is used in order to determine the number of $t \bar{t}$ events. In each channel signal and backgrounds templates are constructed, see Figure11 ATLAS performs a measurement in the lepton+jets channel based on a data sample corresponding to an integrated luminosity of $5.8 \mathrm{fb}^{-1}$ collected at $\sqrt{s}=$ $8 \mathrm{TeV}$, which results in $\sigma_{t \bar{t}}=241 \pm 2$ (stat.) \pm 31 (syst.) \pm 9 (lum.) pb [5].

\subsection{Dileptonic channel}

The $t \bar{t}$ dilepton final states contain either two isolated oppositely charged lepton $(e e, \mu \mu, e \mu)$ or one lepton and one oppositely charged track, missing energy $\left(E_{\mathrm{T}}^{\text {miss }}\right)$ and at least two high $p_{\mathrm{T}}$ jets. The dominant background from Drell-Yan production is estimated from data, while the remaining backgrounds processes, such as single top quark or diboson production, are estimated from Monte Carlo simulation. The cross-section is determined by a cut-and-count method applied on three different samples characterised by events with no $b$-tagged jets, with at least one $b$-tagged jet or with a lepton and an isolated track. The results are then combined by means of a profile likelihood technique which returns $\sigma_{t \bar{t}}=165 \pm 2$ (stat.) \pm 17 (syst.) \pm 3 (lumi) pb at $\sqrt{s}=7 \mathrm{TeV}$ [6]. 


\subsection{All-hadronic channel}

The all hadronic final state is characterized by events with at least six jets, two of which are $b$-tagged. The main source of background is the QCD multi-jet production. Since the background from this source is difficult to simulate correctly, its modelling is based on data. The signal extraction is based on a kinematical fit that exploits the characteristic topology of a hadronic $t \bar{t}$ events: a likelihood maximization is performed with respect to the jet energies while imposing a BreitWigner constraint on the $W$ boson and top quark masses. The measurement is performed on the 2011 data sample corresponding to an integrated luminosity of $4.7 \mathrm{fb}^{-1}$ at $\sqrt{s}=7 \mathrm{TeV}$ and returns $\sigma_{t \bar{t}}=168 \pm 12$ (stat. $)_{-57}^{+60}$ (syst.) \pm 6 (lumi. $) \mathrm{pb} \llbracket 7 \rrbracket$.

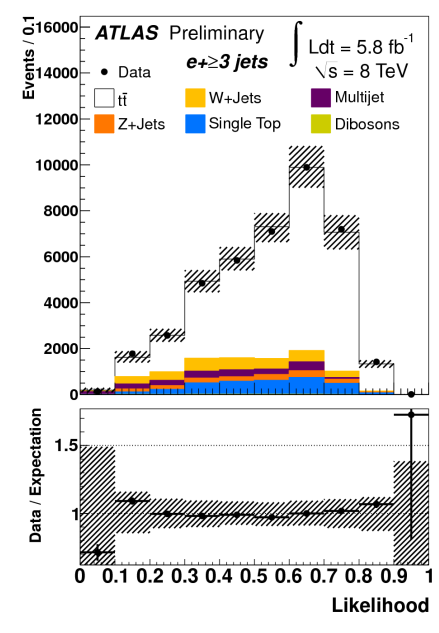

Figure 1: $t \bar{t}$ cross section analysis: fit to the likelihood discriminant distribution in e+jets channel [5].

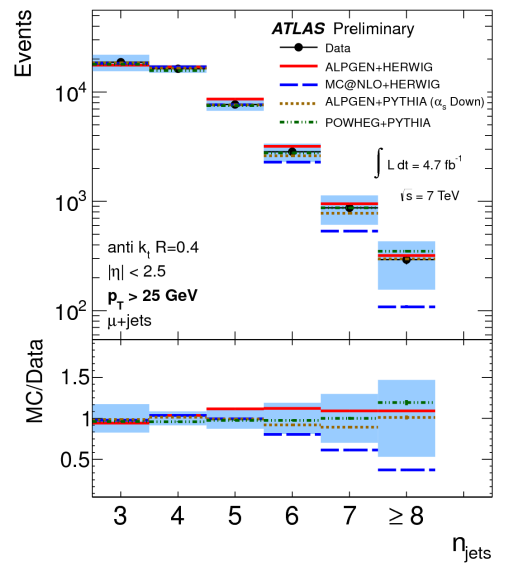

Figure 2: The particle-jet multiplicities for the $\mu$ channel and the jet $p_{\mathrm{T}}$ threshold 25 $\mathrm{GeV}$ [8]: comparison between data and MC predictions.

\subsection{Jet multiplicity in top anti-top final states}

Additional jets in $t \bar{t}$ events are produced through higher order QCD diagrams. Current MC generators model those processes by matrix element calculation or by parton shower approximation. A direct measurement of the jet multiplicity in $t \bar{t}$ events is useful to provide feedback on the modelling of the initial and final state radiation (ISR/FSR) besides testing the predictions of perturbative QCD. ATLAS measured the jet multiplicity in $t \bar{t}$ events in the lepton+jets channel using the full 2011 dataset [8]. The strategy of the analysis is to count the number of jets produced in the events with different jet $p_{\mathrm{T}}$ thresholds: $25,40,60$ and $80 \mathrm{GeV}$. An unfolding procedure is used to correct the jet multiplicity to the parton level. The results indicate that MC@NLO + HERWIG [9, 10] underestimate the events with large multiplicities, suggesting that the MC@NLO jet production is too soft, see Figure 2, POWHEG + PYTHIA [11] 12], ALPGEN + HERWIG [13] and ALPGEN + PYTHIA (with $\alpha_{s}$ - Down variation), on the other hand, are consistent with data in all jet bins. The production of $t \bar{t}$ in association with two extra $b$-tagged jets represents an important background for the production of the Higgs boson in association with $t \bar{t}$, when the Higgs boson decays to $b \bar{b}$. ATLAS performed the measurement of the cross section ratio $R_{H F}$ of $t \bar{t}+H F$ to 
$t \bar{t}+$ jet in the dilepton channel [14]. The fraction of $H F$ jets is extracted by a binned maximum likelihood template fit on the vertex mass distribution. In Figure 3 the extracted value for $R_{H F}$ is shown as a function of the ratio of $t \bar{t}$ events with additional $b$-quarks to $t \bar{t}$ events with additional $c$ or $b$-quarks, $F_{b / H F}$. The result is $R_{H F}=\left[7.1 \pm 1.3(\text { stat. })_{-2.0}^{+5.3}\right.$ (syst.)] \%, consistent at the $1.4 \sigma$ level with LO SM prediction from ALPGEN and at $0.6 \sigma$ level with the approximate NLO result from POWHEG.

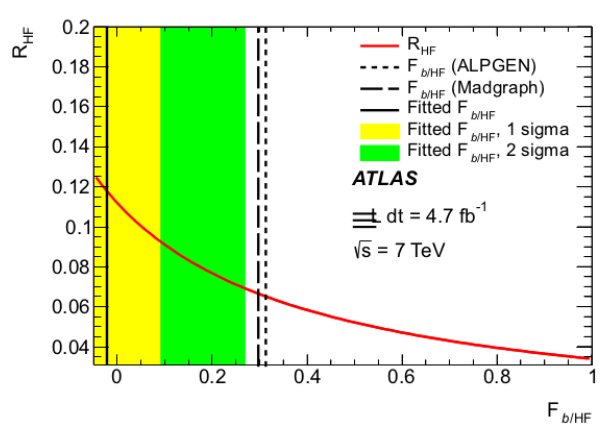

Figure 3: The ratio $R_{H F}$ of fiducial cross sections as a function of $F_{b / H F}$, the ratio of $t \bar{t}$ events with additional $b$-quarks to $t \bar{t}$ events with additional $c$ - or $b$-quarks [14.

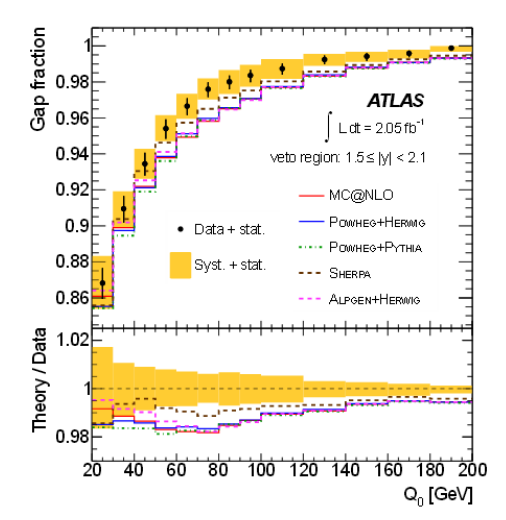

Figure 4: The measured gap fraction as a function of $Q_{0}$ in the $1.5<|y|<2.1$ rapidity regions [15].

\section{$2.5 t \bar{t}$ production with veto on additional central jet activity}

ATLAS performed a measurement of the jet activity arising from the QCD emission in $t \bar{t}$ events using $2.05 \mathrm{fb}^{-1}$ of data in the dilepton channel [15]. Events are selected with two opposite sign leptons $(e / \mu)$, missing transverse energy and two $b$-tagged jets. The strategy relies on the so-called gap fraction, defined as $f\left(Q_{0}\right)=\frac{\sigma\left(Q_{0}\right)}{\sigma}$, where $\sigma$ is the total $t \bar{t}$ cross section and $\sigma\left(Q_{0}\right)$ is the cross section for $t \bar{t}$ events with no additional jets in a given rapidity ${ }^{1}$ region and $p_{\mathrm{T}}>Q_{0}$. The behaviour observed in data is then compared to various MC generators. The MC generators provide a reasonable description of the results obtained from data when the additional jets are vetoed in the central rapidity range $(|y|<0.8)$; however there is a clear overstimation of the jet activity in the most forward region $(1.5<|y|<2.1)$, see Figure 4

\section{Single top quark production}

Single top production is a sensitive probe for a number of new physics models and it can also be used for direct measurements of the CKM matrix element $\left|V_{t b}\right|$. The cross-section measurements have been performed in the $t-, W t-, s$-channels.

\section{1 $t$-channel}

Single top cross section in the $t$-channel has been measured on $\sqrt{s}=7$ [16] and $8 \mathrm{TeV}$ [17] $p p$ collisions. The selected events contain one charged lepton, missing transverse momentum,

\footnotetext{
${ }^{1}$ Rapidity is defined as $y=0.5 \ln \left[\left(E+p_{z}\right) /\left(E-p_{z}\right)\right]$, where $E$ denotes the energy and $p_{z}$ is the component of the momentum along the beam axis.
} 
and two or three jets, one of which is $b$-tagged. The main backgrounds are multi-jet, $W+$ jets and $t \bar{t}$ events. Data are classified according to lepton flavour, number of jets and $b$-tags: two or three jets, with one $b$-tagged jets. A neural network (NN) discriminant is built to separate the signal from the background. The top and anti-top cross section are extracted by mean of a binned maximum likelihood fit on the $\mathrm{NN}$ output distribution. A summary of the cross section results at $\sqrt{s}=7$ and $8 \mathrm{TeV}$ is showed in Figure 5. Then the ratio $R_{t}=\frac{\sigma_{t}}{\sigma_{\bar{t}}}$ is estimated obtaining a result of $R_{t}=1.81 \pm 0.10$ (stat. $)_{-0.20}^{+0.21}$ (syst.) pb on an integrated luminosity of $4.7 \mathrm{fb}^{-1}$ at $\sqrt{s}=7 \mathrm{TeV}$ [16]. The coupling strength at the $W-t-b$ vertex has been estimated in $\left|V_{t b}\right|=1.04_{-0.11}^{+0.10}$ on an integrated luminosity of $5.8 \mathrm{fb}^{-1}$ at $\sqrt{s}=8 \mathrm{TeV}$ and constrained to be greater than 0.80 at the $95 \%$ confidence level [17].

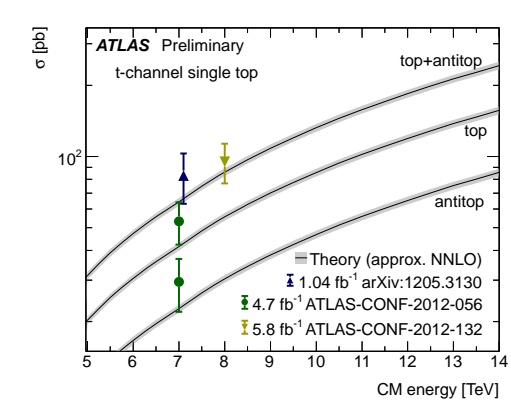

Figure 5: Summary of ATLAS $\sqrt{s}=7 \mathrm{TeV}$ and $\sqrt{s}=8 \mathrm{TeV} t$-channel single top measurements [17.

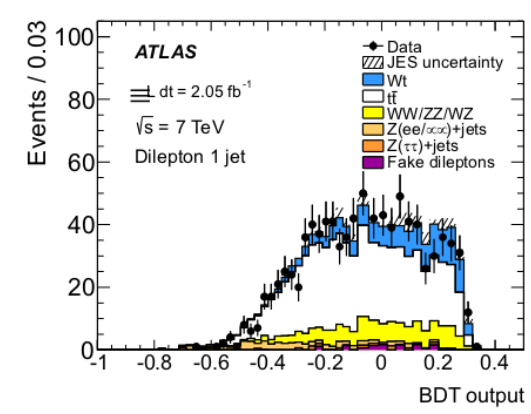

Figure 6: $W t$-channel single top cross section measurement: Boosted Decision Tree output for selected events in 1-jet [18].

\subsection{Wt -channel}

The cross section measurement in the $W t$-channel is performed in a dilepton dataset corresponding to an integrated luminosity of $2.05 \mathrm{fb}^{-1}$ at $\sqrt{s}=7 \mathrm{TeV}$. A Boosted Decision Tree (BDT) was implemented to improve the discrimination between the $W t$ signal and $t \bar{t}$ production. The measured cross section is extracted using a likelihood fit on the BDT output, see Figure 6. An evidence of the $W t$ production was seen with a observed significance of $3.3 \sigma$ ( $3.4 \sigma$ expected). The cross section is $\sigma(W t)=16.8 \pm 2.9$ (stat) \pm 4.9 (syst) pb and the CKM matrix element $V_{t b}$ is measured to be $\left|V_{t b}\right|=1.03_{-0.19}^{+0.16}[18]$.

\section{Conclusion}

The data taking in 2011 and 2012 at $\sqrt{s}=7$ and $8 \mathrm{TeV}$ at the LHC has been very successful. The ATLAS experiment recorded up to $25 \mathrm{fb}^{-1}$ of $p p$ collisions. The measured top pair cross section in various final states is found to be in agreement with the SM expectation. Single top cross section results in the $t$ - and $W t$-channel are also reported. Furthermore both channels provide consistent measurements of the $W-t-b$ vertex coupling. 


\section{References}

[1] F. Abe et al., "Observation of top quark production in $\bar{p} p$ collisions," Phys.Rev.Lett., vol. 74, pp. 2626-2631, 1995.

[2] S. Abachi et al., "Observation of the top quark," Phys.Rev.Lett., vol. 74, pp. 2632-2637, 1995.

[3] M. Czakon, P. Fiedler, and A. Mitov, "The total top quark pair production cross-section at hadron colliders through $o\left(\alpha_{s}^{4}\right)$, , arXiv:1303.6254, 2013.

[4] ATLAS Collaboration, "The ATLAS Experiment at the CERN Large Hadron Collider," JINST, vol. 3, p. S08003, 2008.

[5] ATLAS Collaboration, "Measurement of the top quark pair production cross section in the single-lepton channel with ATLAS in proton-proton collisions at $8 \mathrm{TeV}$ using kinematic fits with b-tagging," ATLAS-CONF-2012-149, 2012. https://cds.cern.ch/record/1493488.

[6] ATLAS Collaboration, "Measurement of the cross section for top-quark pair production in $p p$ collisions at $\sqrt{s}=7 \mathrm{TeV}$ with the ATLAS detector using final states with two high- $p_{\mathrm{T}}$ leptons," JHEP, vol. 1205, p. 059, 2012.

[7] ATLAS Collaboration, "Measurement of the $t \bar{t}$ production cross section in the all-hadronic channel in ATLAS with $\sqrt{s}=7$ TeV data," ATLAS-CONF-2012-031, 2012. https://cds.cern.ch/record/1432196.

[8] ATLAS Collaboration, "Measurement of the jet multiplicity in top anti-top final states produced in 7 TeV proton-proton collisions with the ATLAS detector," ATLAS-CONF-2012-155, 2012. https://cds.cern.ch/record/1493494.

[9] S. Frixione and B. R. Webber, "Matching NLO QCD computations and parton shower simulations," JHEP, vol. 0206, p. 029, 2002.

[10] G. Corcella, I. Knowles, G. Marchesini, S. Moretti, K. Odagiri, et al., "HERWIG 6: An Event generator for hadron emission reactions with interfering gluons (including supersymmetric processes)," JHEP, vol. 0101, p. 010, 2001.

[11] S. Alioli, P. Nason, C. Oleari, and E. Re, "A general framework for implementing NLO calculations in shower Monte Carlo programs: the POWHEG BOX,” JHEP, vol. 1006, p. 043, 2010.

[12] T. Sjostrand, S. Mrenna, and P. Z. Skands, "PYTHIA 6.4 Physics and Manual," JHEP, vol. 0605, p. 026, 2006.

[13] M. L. Mangano, M. Moretti, F. Piccinini, R. Pittau, and A. D. Polosa, “ALPGEN, a generator for hard multiparton processes in hadronic collisions,” JHEP, vol. 0307, p. 001, 2003.

[14] ATLAS Collaboration, "A study of heavy flavor quarks produced in association with top quark pairs at $\sqrt{s}=7 \mathrm{TeV}$ using the ATLAS detector," arXiv:1304.6386, 2013.

[15] ATLAS Collaboration, "Measurement of $t \bar{t}$ production with a veto on additional central jet activity in pp collisions at $\sqrt{ }(s)=7 \mathrm{TeV}$ using the ATLAS detector," Eur.Phys.J., vol. C72, p. 2043, 2012.

[16] ATLAS Collaboration, "Measurement of the $t$-channel single top-quark and top-antiquark production cross-sections and their ratio in pp collisions at $\sqrt{s}=7 \mathrm{TeV}$," ATLAS-CONF-2012-056, 2012. https://cds.cern.ch/record/1453783.

[17] ATLAS Collaboration, "Measurement of t-channel single top-quark production in pp collisions at $\sqrt{(s)}=8 \mathrm{TeV}$ with the ATLAS detector," ATLAS-CONF-2012-132, 2012. https://cds.cern.ch/record/1478371.

[18] ATLAS Collaboration, "Evidence for the associated production of a $W$ boson and a top quark in ATLAS at $\sqrt{s}=7$ TeV," Phys.Lett., vol. B716, pp. 142-159, 2012. 